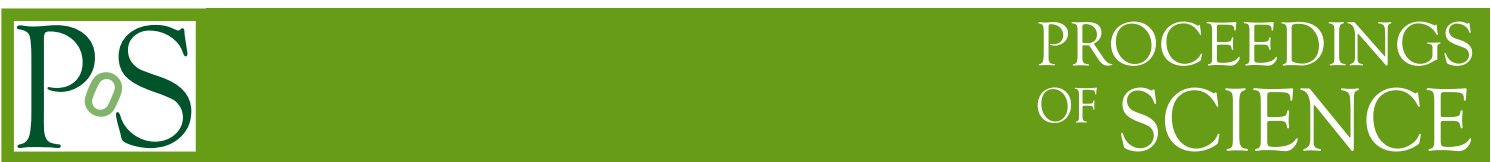

\title{
Eigenvector space of narrow-line Seyfert 1 galaxies
}

\author{
Dawei $\mathbf{X u}^{*}$ \\ National Astronomical Observatories, Chinese Academy of Sciences, 20A Datun Road, Beijing \\ 100012, China \\ E-mail: dwxu@nao.cas.cn
}

\section{S. Komossa}

Max-Planck-Institut für Radioastronomie, Auf dem Hügel 69, D-53121 Bonn, Germany

\begin{abstract}
We have performed an analysis of the correlation space of narrow- and broad-line Seyfert 1 galaxies, in order to identify main drivers of their intriguing emission-line and continuum properties. In particular, we paid attention to the density of the narrow-line region. A principal component analysis then shows that the density is a key ingredient of the Eigenvector 1 space of our sample, as important as the Eddington ratio. Our finding implies a close link between the properties of the central engine and the host galaxy.
\end{abstract}

Nuclei of Seyfert galaxies and QSOs - central engine and conditions of star formation November 6-8, 2012

Max-Planck-Insitut für Radioastronomie (MPIfR), Bonn, Germany

${ }^{*}$ Speaker. 


\section{Introduction}

Narrow-line Seyfert 1 (NLS1) galaxies are a population of active galactic nuclei (AGN) which are characterized by narrow Balmer lines from the broad-line region (BLR), strong Fe II emission and weak [O III] emission. They stand out in the AGN correlation space by clustering at one extreme end (e.g., [1], [2]). They therefore provide us with constraints on models of black hole growth and AGN evolution (see [9] for a review).

Principal component analysis (PCA) is a useful tool to uncover the strongest correlations among a set of object properties. While not without shortcomings (see, e.g., [3] for a critical discussion), it provides some information on the underlying physical drivers behind the observed correlations. We have applied it to a sample of nearby NLS1 and broad-line Seyfert 1 (BLS1) galaxies, with the aim of uncovering the major drivers of their emission-line and continuum properties.

The galaxy sample was first introduced by [11], and consists of $\sim 100$ narrow- and broad-line AGN which have Sloan Digital Sky Survey (SDSS) spectra available. Our main focus was on the NLS1 galaxies, while the BLS1 galaxies serve as a comparison sample. The broad-line widths of our sample range between FWHM(H $\beta) \sim 1070 \mathrm{~km} \mathrm{~s}^{-1}$ and $6200 \mathrm{~km} \mathrm{~s}^{-1}$. We have set the "dividing line" between NLS1 and BLS1 galaxies at $2000 \mathrm{~km} \mathrm{~s}^{-1}$, following historical convention (e.g., [5]), but any other FWHM value can in principle be imposed on our sample.

This work (see also [12]) is the fourth in a sequence, in a study devoted to NLS1 galaxies. The first paper reported on the difference in the density of the narrow-line region (NLR) of NLS1 and BLS1 galaxies ([11]), the second focussed on the locus of NLS1 galaxies on the $M-\sigma$ plane ([7]), and the third addressed a subgroup of NLS1 galaxies which show extreme emission-line outflows ([9]).

\section{Results}

Our main results can be summarized as follows:

- As previously shown for other NLS1 samples (e.g., [6]), and applying the common scaling relations (e.g., review by [10]), we confirm that NLS1 galaxies, as a class, are characterized by smaller black hole masses, and higher Eddington ratios than their BLS1 counterparts. Figure 1 shows the distribution of black hole masses and Eddington ratios of our sample.

- We have run a PCA, based on the following parameters which were derived for our sample: the SDSS $i$ band magnitude, the FWHM of the broad component of $\mathrm{H} \beta$, the ratio of total [O III] $\lambda 5007$ over total $\mathrm{H} \beta$ emission, the ratio of Fe II $\lambda 4570$ over total $\mathrm{H} \beta$ emission, the FWHM of [S II], the velocity shift of the core of [O III] (with respect to [S II]), and the intensity ratio of $[S \mathrm{II}] \lambda 6716 / \lambda 6731$. The latter directly provides a measurement of the NLR density. Each parameter provides independent information. Based on this approach, we have found, that the NLR density is a key parameter of the Eigenvector 1 of our sample. It turns out to be as important as the Eddington ratio (Figure 2).

- Eigenvector 2 is highly related to luminosity. NLS1 and BLS1 galaxies are well distinguished in EV1 space, while they are merged in EV2 space. 

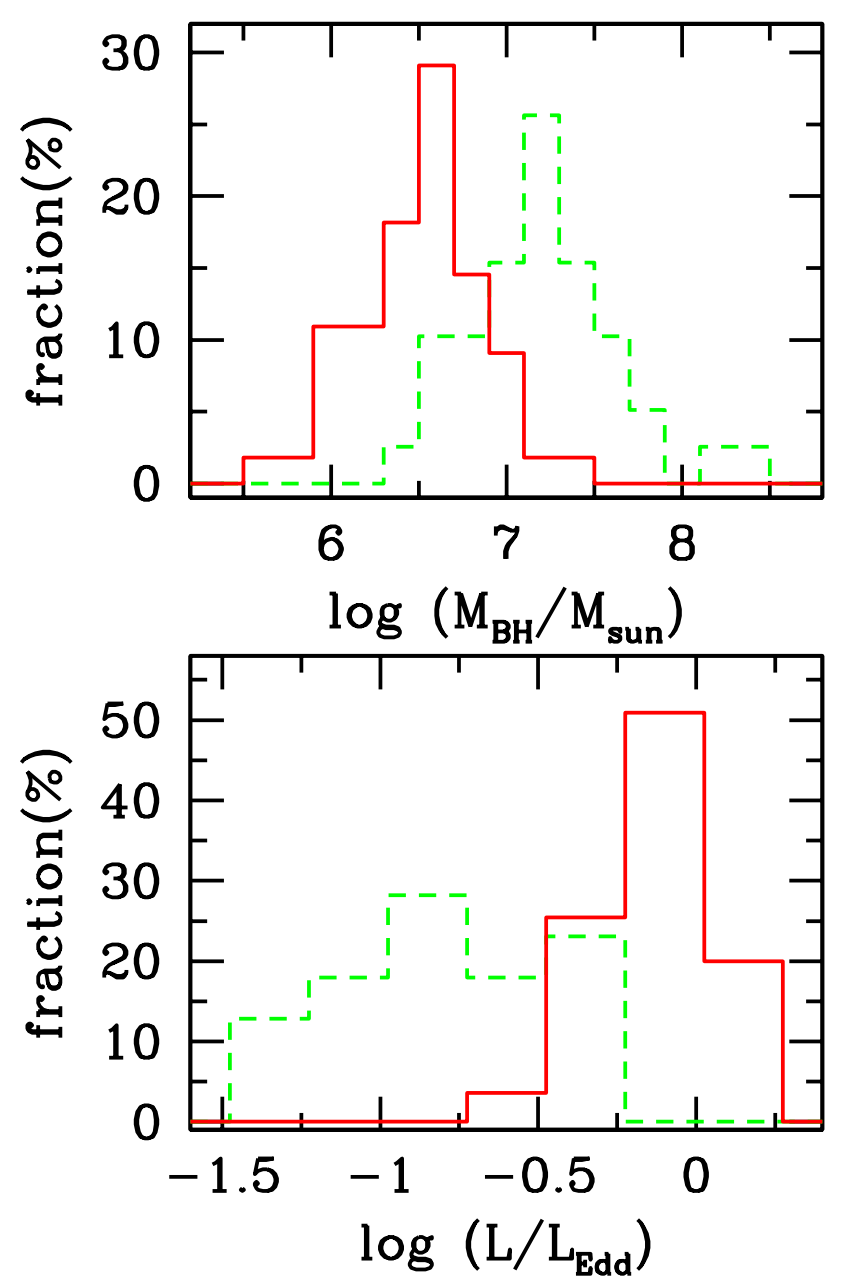

Figure 1: Distribution of black hole masses and Eddington ratios (solid line: NLS1 galaxies, dashed line: BLS1 galaxies).

- Several NLS1 galaxies with extreme blueshifts of [OIII] (so called "blue outliers") fall into the corner of high Eddington ratio and high luminosity in the EV1-EV2 diagram (Figure 3). They share this location with broad absorption line quasars (e.g., [2]). This fact might hint at possible links between these two subclasses of AGN, which both show observational evidence for the occurence of strong outflows, albeit at different scales.

\section{Discussion and implications}

We have shown that the NLR density is a significant parameter in EV1 space, and, in fact, as important as the Eddington ratio. This finding establishes the density as a key ingredient, when aiming at understanding the multi-wavelength correlation properties of NLS1 galaxies.

Adding density to EV1 space was of particular importance, because the NLR density is representative of the interstellar medium of the host galaxy. Our findings therefore imply a close link between the central engine properties and the host galaxies. 


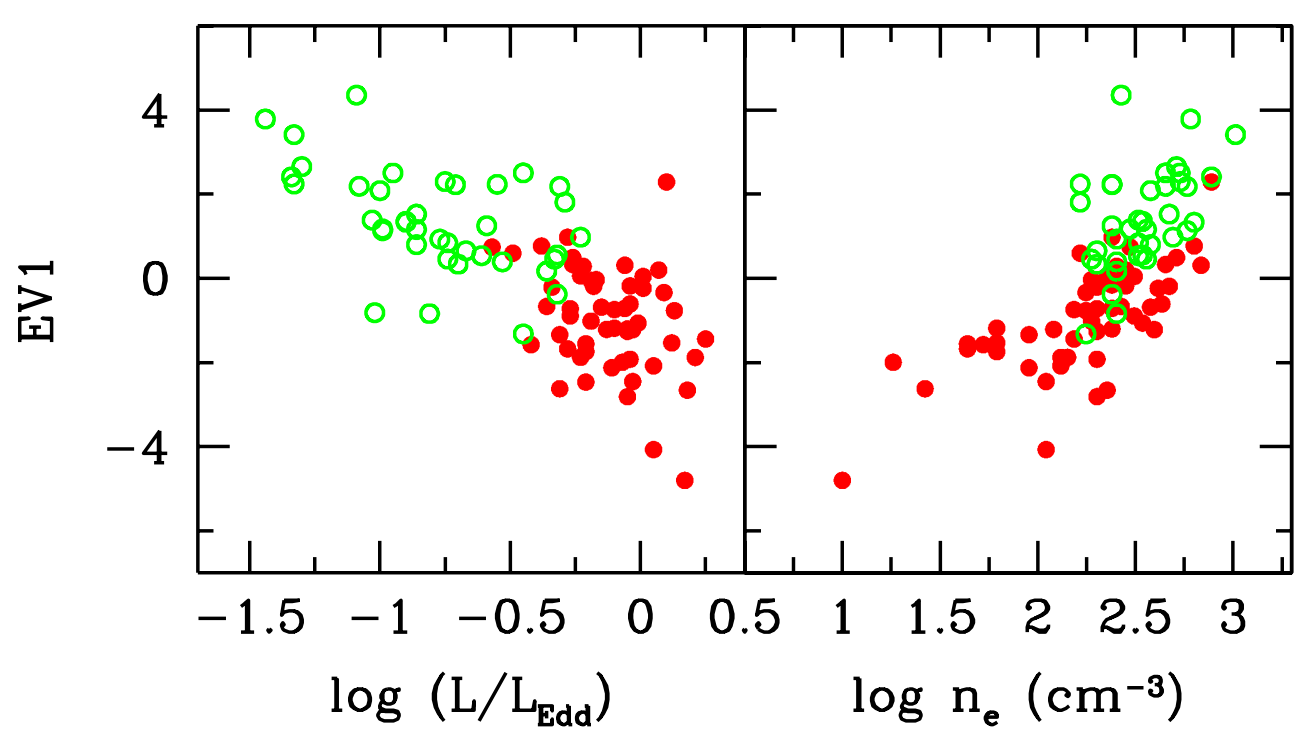

Figure 2: Correlations of EV1 with the NLR density $n_{\mathrm{e}}$ and the Eddington ratio $L / L_{\mathrm{Edd}}$. (filled circles: NLS1 galaxies, open circles: BLS1 galaxies).

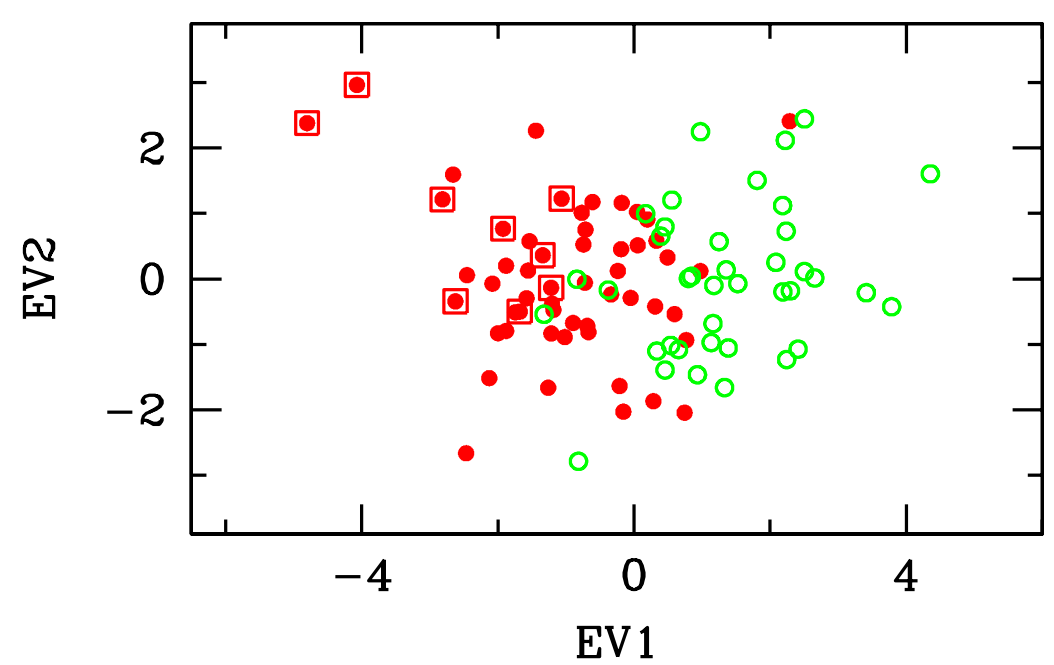

Figure 3: Distribution of NLS1 galaxies (filled circles) and BLS1 galaxies (open circles) of our sample in the EV1-EV2 space. [O III] blue outliers are marked with an extra open square.

Such a link is potentially expected, on the one hand, when winds or outflows are at work, or, on the other hand, might also be caused by bar-driven inflows.

Winds and outflows are particularly strong in NLS1 galaxies (e.g.,[9]), and might be linked to the high Eddington ratios in NLS1 galaxies. Whether accretion-driven outflows may propagate up into the NLR is currently being explored. We also note that galaxy merger simulations predict strong outflows, but so far, we do not see a strong excess of mergers among our NLS1 galaxies, when compared to BLS1 galaxies (but note that few of the NLS1 galaxies have high-quality host images). 
Finally, we note that recent studies have shown that NLS1 galaxies have a higher bar fraction than BLS1 galaxies (e.g., [4]). Since bars are efficient in transporting gas inward, they might supply the NLR with (low-density) gas, thus providing another possible explanation for the NLR properties of NLS1 galaxies. Bar-driven instabilities can also lead to pseudo-bulges by internal secular processes; and several lines of evidence have been presented in recent years, that secular processes indeed play a role in NLS1 galaxies.

In summary, NLS1 galaxies are important targets for our understanding of black hole growth and evolution, and of issues of feeding and feedback.

A number of future follow-up studies suggest themselves, including: (1) Selection of larger NLS1 samples from the latest SDSS data releases which, in particular, have all their classical emission lines detected. This ensures that emission-line diagnostics can be performed the same way as it was done for our current sample (like, for instance, using the [S II] $\lambda 6716 / \lambda 6731$ emission-line ratio for density diagnostics). (2) Obtaining high-quality host images of the galaxies of our sample, in order to measure their host type, and host properties, and ultimately add host properties to the correlation analyses. (3) Nearest-neighbor analyses of larger NLS1 samples, in order to investigate if NLS1 and BLS1 galaxies reside in similar large-scale environments.

\section{Acknowledgments}

This work is supported by the National Natural Science Foundation of China (Grant No. 11273027) and the National Basic Research Program of China, referred to as 973 program (Grant No. 2009CB824800). SK would like to thank the Aspen Center for Physics for their hospitality. The Aspen Center for Physics is supported by NSF Grant No. 1066293. This research has made use of the SDSS data base, and of the NASA/IPAC Extragalactic Database (NED) which is operated by the Jet Propulsion Laboratory, California Institute of Technology, under contract with the National Aeronautics and Space Administration. Funding for the SDSS and SDSS-II has been provided by the Alfred P. Sloan Foundation, the Participating Institutions, the National Science Foundation, the U.S. Department of Energy, the National Aeronautics and Space Administration, the Japanese Monbukagakusho, the Max Planck Society, and the Higher Education Funding Council for England. The SDSS Web Site is http://www.sdss.org/. The SDSS is managed by the Astrophysical Research Consortium for the Participating Institutions. The Participating Institutions are the American Museum of Natural History, Astrophysical Institute Potsdam, University of Basel, University of Cambridge, Case Western Reserve University, University of Chicago, Drexel University, Fermilab, the Institute for Advanced Study, the Japan Participation Group, Johns Hopkins University, the Joint Institute for Nuclear Astrophysics, the Kavli Institute for Particle Astrophysics and Cosmology, the Korean Scientist Group, the Chinese Academy of Sciences (LAMOST), Los Alamos National Laboratory, the Max-Planck-Institute for Astronomy (MPIA), the Max-PlanckInstitute for Astrophysics (MPA), New Mexico State University, Ohio State University, University of Pittsburgh, University of Portsmouth, Princeton University, the United States Naval Observatory, and the University of Washington. 


\section{References}

[1] T. A. Boroson \& R. F. Green: The emission-line properties of low-redshift quasi-stellar objects, ApJS 80 (1992) 109.

[2] T. A. Boroson: Black hole mass and Eddington ratio as drivers for the observable properties of radio-loud and radio-quiet QSOs, ApJ 565 (2002) 78.

[3] T. A. Boroson: Principal component analysis of QSO properties, In: Proceedings of ASP Conference Series 311 (2004) 3.

[4] D. M. Crenshaw, S. B. Kraemer \& J. R. Gabel: The host galaxies of narrow-Line Seyfert 1 galaxies: evidence for bar-driven fueling, AJ 126 (2003) 1690.

[5] R. W. Goodrich: Spectropolarimetry of 'narrow-line' Seyfert 1 galaxies, ApJ 342 (1989) 224.

[6] D. Grupe: A complete sample of soft X-ray-selected AGNs. II. statistical analysis, AJ 127 (2004) 1799.

[7] S. Komossa, \& D. Xu: Narrow-line Seyfert 1 galaxies and the $M_{\mathrm{BH}}-\sigma$ relation, ApJ 667 (2007) L33.

[8] S. Komossa: Narrow-line Seyfert 1 galaxies, RevMexAA (Serie de Conferencecias) 32 (2008) 86.

[9] S. Komossa, D. Xu, H. Zhou, et al.: On the nature of Seyfert galaxies with high [O III] $\lambda 5007$ blueshifts, ApJ 680 (2008) 926.

[10] B. M. Peterson: Masses of black holes in active galactic nuclei: implications for narrow-line Seyfert 1 galaxies, In: Proceedings of the Workshop Narrow-Line Seyfert 1 Galaxies and Their Place in the Universe, PoS (NLS1) 032 (2011).

[11] D. Xu, S. Komossa, H. Zhou, et al.: The narrow-line region of narrow-line and broad-line type 1 active galactic nuclei. I. A zone of avoidance in density, ApJ 670 (2007) 60.

[12] D. Xu, S. Komossa., H. Zhou, et al.: Correlation Analysis of a large sample of narrow-line Seyfert 1 galaxies: linking central engine and host properties, AJ 143 (2012) 83. 